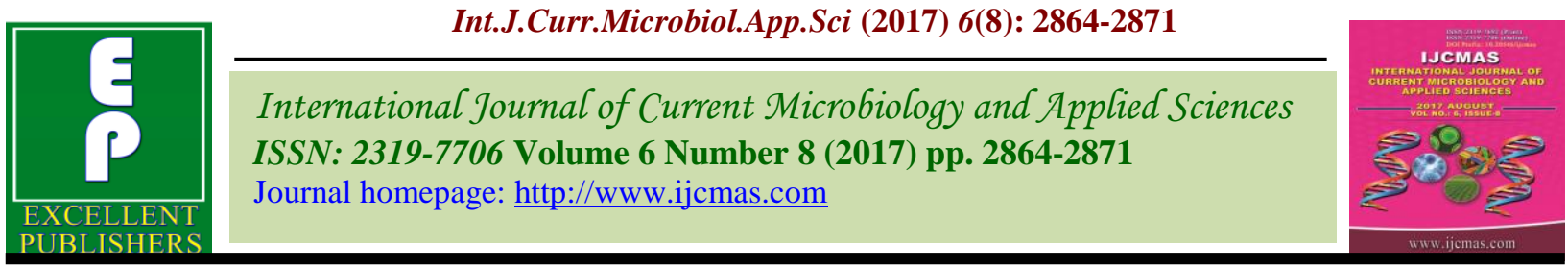

Original Research Article https://doi.org/10.20546/ijcmas.2017.608.342

\title{
Effect of Intensity of Heating on Qualitative Tests for the Detection of Common Adulterants in Milk
}

\author{
Chaudhari Priyankaben Rameshbhai, Kakade Pooja Vilasrao, Jayswal Dharin, \\ S.I. Patel, A.I. Shaikh" ${ }^{*}$ and K.D. Aparnathi \\ *Corresponding author
}

\section{A B S T R A C T}

Keywords

Milk, Adulteration, Intensity of heating, Glucose, Sucrose,

Ammonium

sulphate, Formalin.

Article Info

Accepted:

23 June 2017

Available Online:

10 August 2017
Qualitative tests for the adulterant detection are advantageous because of it being selective in nature. Because of procedural variations in these tests, the inferences drawn when these tests are used remains doubtful sometimes. One of such sources of variation, intensity of heating in some tests for detection of glucose, sucrose, ammonium sulphate and formalin were evaluated. The optimum period of heating was found to be 3 minutes for Barfoed test for glucose, 5 minutes for Seliwanoff's test for sucrose, 20 seconds for Phenol test for ammonium sulphate, 1 minute for Leach test using direct heating and 4 minutes for heating on boiling water bath for formalin.

\section{Introduction}

India is world's highest milk producer with milk production of155.5 Million Metric Tonnes (Anon., 2017).However, only 20-22 per cent milk is handled by the organized sector as compared to 70 per cent of world average (Srivastava, 2010). As large proportion of milk produced is processed by unorganized sector, the quality of such milk and milk products is far from satisfactory (Barui et al., 2013). Chemical quality of milk is linked with the hidden desire of unscrupulous milk producers/suppliers for making profit (Arora et al., 2004 and Souza et al., 2011).The practice of adulteration of milk is as old as history and is one of the major problems that stands against the progress of dairying in India with respect to quality requirements for the international market which ultimately affects the export of dairy products. Analytical methods has been developed for most of the adulterants but venal traders find more innovative ways to adulterate the milk with cheaper ingredients (Srivastava, 2010 and Sharma et al., 2012). Recent reports have revealed the use of urea, glucose, sucrose, maltodextrin, starch, water, salt, gelatin and detergent for the purpose of adulterating milk (Srivastava, 2010).

Several test kits, instrumental methods, physical methods and chemical qualitative tests are used for the detection of adulterants in milk (Kamthania et al., 2014). Chemical methods, having the advantage of convenience, rapid and cost effective, are widely used for the detection of adulterants in milk. Qualitative tests utilizing various reagents have been developed for the 
detection of ammonium sulphate (phenol test), glucose (Barfoed's test), sucrose (resorcinol test) and formaldehyde (Leach test).Many of these qualitative tests have procedural various in terms of intensity of hating involved in these tests. Such variations can lead to either false positive or false negative results in qualitative tests for detergents with serious consequences for dairy plants.

Heating is a common phase involved in several qualitative tests used for the detection of adulterants in milk. However, tests results may vary depending on intensity of heating where the methods include heating. Therefore, the present work is carried out to study the effect of intensity of heating on qualitative tests used for the detection of common adulterants in milk.

\section{Materials and Methods}

\section{Milk sample}

Raw milk samples were procured from dairy farm of Anand Agricultural University, Anand. Milk samples were prepared by spiking the raw milk with adulterants at the suitable level for a particular test.

\section{Chemicals and reagents}

Ammonium molybdate (Qualigens Fine Chemicals Ltd., Mumbai), Cupric acetate (Qualigens Fine Chemicals Ltd., Mumbai), Ferric chloride (Qualigens Fine Chemicals Ltd., Mumbai), Formalin (SD Fine Chemicals Ltd., Mumbai), Hydrochloric acid (Qualigens Fine Chemicals Ltd., Mumbai), Lactic acid (Loba-Chemie Pvt. Ltd., Mumbai), Sodium hypochlorite (SD Fine Chemicals Ltd., Mumbai), Phenol (Spectrochem Pvt. Ltd., Vadodara), Phosphoric acid (Spectrochem Pvt. Ltd., Vadodara), Resorcinol (Qualigens fine chemicals), Sodium hydroxide
(Qualigens Fine Chemicals Ltd., Sodium tungstate (Loba-chemie Pvt. Ltd., Mumbai).

Test procedures used for detection of adulterants in milk

\section{Barfoed's test}

Barfoed's test was performed as per the procedure given by Roy and Mittal (1977). In a test tube one ml of milk sample was taken. Then, $1 \mathrm{ml}$ of modified Barfoed's reagent was added to the test tube containing milk sample and heated in a boiling water bath for period of heating varying from 1, 2, 3, 4 and 5 minutes. After removing from the water bath, tests tubes were cooled to room temperature and $1 \mathrm{ml}$ of phosphomolybdic acid reagent was added. Content was mixed and change in colour was observed. Presence of glucose was confirmed by development of deep blue colour, whereas, control sample gave a pale blue.

\section{Seliwanoff test}

Addition of sucrose in milk was evaluated by Seliwanoff test (Srivastava, 2010). In a test tube $3 \mathrm{ml}$ of milk sample was taken. Subsequently, $5 \mathrm{ml}$ of Seliwanoff reagent $(0.05 \%)$ was added in the test tube. Contents were then mixed well and the test tubes were placed in a boiling water bath for variable period of 1, 3, 5, 7 and 9 minutes. Presence of sucrose in the sample was confirmed by appearance of red colour, whereas control samplegave faint red colour.

\section{Phenol test (for detection of ammonium sulphate)}

Ammonium sulphate in milk was determined using the method developed by Mittal and Roy (1976). In a test tube $1 \mathrm{ml}$ of suspected milk was taken. Then, $0.5 \mathrm{ml}$ of sodium hydroxide $(2 \%), \quad 0.5 \mathrm{ml}$ of sodium 
hypochlorite (2\%) and $0.5 \mathrm{ml}$ of phenol solution $(5 \%)$ were added. Contents were mixed properly and heated for variable period of $5,10,15,20,25$ and 30 seconds in boiling water bath. Appearance of deep blue colour indicates presence of ammonium sulphate, however, development of salmon pink colour or faint blue colour indicates that the sample is free from ammonium sulphate.

\section{Leach test}

Formaldehyde addition was detected by Leach test using the method given by Williams and Sherman (1905). In a test tube5 $\mathrm{ml}$ of milk sample was mixed with an equal volume of concentrated hydrochloric acid containing $1 \mathrm{ml}$ of ferric chloride solution to each $500 \mathrm{ml}$ of acid. The sample was heated directly over a flame for variable period $30 \mathrm{~s}$, $1 \mathrm{~min} 30 \mathrm{~s}, 2 \min 30 \mathrm{~s}, 2 \mathrm{~min}, 2 \min 30 \mathrm{~s}, 3$ min. Test tube was rotated to break up the curd. A violet colour indicates the presence of formaldehyde.

While, for checking the effect of intensity of heating in water bath method from Sharma (2012) was used. The test tubes were heated for variable periods of 2, 4, 6, 8 and 10 minutes in boiling water bath.

\section{Results and Discussion}

Qualitative tests are based on chemical reactions between the reagents used and adulterants added. Moreover, these reactions are affected by temperature as it plays an important role in solubility and ionization of compounds (Filion et al., 2007).

The variation in temperature may further affect the performance of qualitative tests employed for detection of adulterants in milk. Therefore effect of temperature on performance of qualitative test for detection of adulterants in milk was evaluated.
Some qualitative test involved heating of sample for detection of adulterant in milk. The variation in method of heating (direct on flame or in boiling water bath) and duration of heating have been reported in present study and evaluated for the effect of the temperature on the performance of the test.

\section{Effect of temperature on Barfoed's test for detection of glucose in milk}

Barfoed's test used for detection of glucose in milk involves heating as one of its procedural step. Hence, this test was evaluated for effect of duration of heating on test performance. Barfoed's test was evaluated for the intensity of heating of milk on detection of glucose using the milk samples spiked with 0.1 per cent glucose. Heating intervals selected were $0,1,2,3,4$ and 5 minutes.

It can be observed from the results obtained in figure 1, that before initiating the heating, faint blue colour was obtained both in control as well as in adulterated sample and there was no change in colour in both the cases upon heating up to 1 minute. On further heating in boiling water bath difference in intensity of the colour in control sample and adulterated sample was noticed. Heating interval of 2 minutes gave a slight increase in intensity of blue colour in sample containing glucose but can fairly distinguish thecontrol sample. However, the obvious difference in colour intensity was observed at the heating interval of 3 minutes, where, the colour of the test tube containing sample was intense blue and that in case of the control sample was light blue. However, further heating caused the colour of the adulterated sample to become darker, for both the sample as well as control.

The optimum difference in colour intensity between the control sample and that of the adulterated sample was found at heating interval of 3 minutes and henceforth, this time 
interval for heating can be used for the detection of added glucose in milk by using Barfoed's test. Moreover, the heating time of 3 minutes for Barfoed's test is also suggested by many author's (Roy and Mittal, 1977; DGHS, 2005; Vishweshwar and Krishnaiah, 2005; NDDB, 2009; Srivastava, 2010 and FSSAI, 2012).

\section{Effect of temperature on Seliwanoff test for detection of sucrose in milk}

As heating was involved in Seliwanoff test used for detection of sucrose in milk, effect of duration of heating on performance of the test was evaluated. Milk samples were prepared by addition of sucrose at 0.1 per cent and the test was carried outby heating in a boiling water bath for different duration of heating ( 0 , 1, 3, 5, 7 and 9 minutes). Seliwanofftest was studied to evaluate the intensity of heating on detection of sucrose in milk and results obtained are presented in figure 2 .

During performing the test it was observed that before heating in boiling water bath there was no colouration in the control as well as in adulterated sample. Similar result was observed when the test tubes were heated for up to 1 minute. On further heating (3 minutes), difference in intensity of colour of the control and adulterated sample was observed but was not significant enough to distinguish between the control and adulterate sample. The definite difference in colour intensity was detected upon heating the samples up to 5 minutes. Upon further heating the colour of the adulterated sample became slight darker, however the colour of the control sample also becoming darker in such a way that difference in colour intensity of control sample and that of the adulterated sample became narrow.

The maximum difference in colour intensity between the control sample and that of the adulterated sample was observed at heating interval of 5 minutes. Therefore, heating period of 5 minutes can be considered optimum for performing the Seliwanoff test. Heating period of 5 minutes for performing Seliwanoff test is also suggested by NDDB (2009), Srivastava (2010), FSSAI (2012) and Sharma et al., (2012).

\section{Effect of heating duration on phenol test for detection of ammonium sulphate}

As heating was involved in phenol test used for detection of ammonium sulphate in milk, effect of duration of heating on performance of the test was evaluated. Milk samples were prepared by addition of ammonium sulphate at 0.01 per cent and the phenol test was carried outby heating in a boiling water bath for different heating intervals $(0,5,10,15,20$, 25 and 30 seconds) and results obtained are presented in figure 3 .

During performing the test it was observed that before initiation of heating the blue colour was obtained both in control as well as in adulterated sample. On heating in boiling water bath up to 15 seconds no change in colour was observed either in control or in adulterated sample. On further heating up to 20 seconds control sample turns yellow in colour and the colour in adulterated sample remained blue. Upon further heating up to 25 seconds result obtained remained unaffected i.e. similar to result of heating up to 20 seconds. However, in case of heating up to 30 seconds colour of control remained orange but colour of adulterated sample faded slightly. Heating further up to 45 seconds the colour of adulterated sample gradually faded and on heating of 60 seconds, colour of adulterated sample turned yellowish, which was similar to that of the control sample. Therefore, heating period up to 20 seconds can be recommended for the detection of ammonium sulphate in milk using phenol test. 
Fig.1 Effect of heating duration on Barfoed (Performed in milk) for Glucose

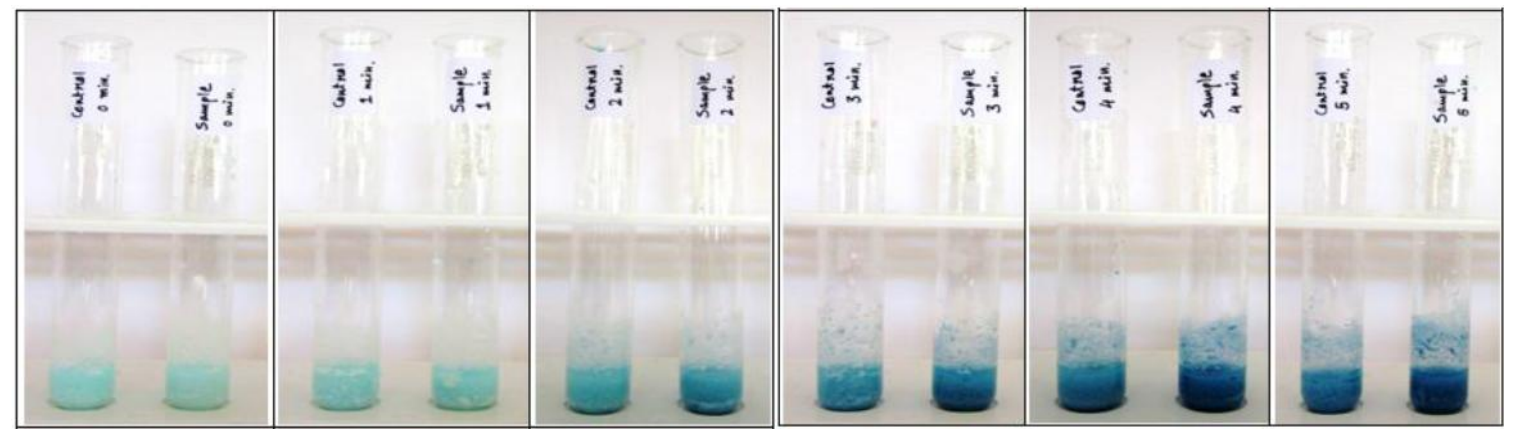

Fig.2 Effect of heating duration on Seliwanoff test (Resorcinol solution) for Sucrose

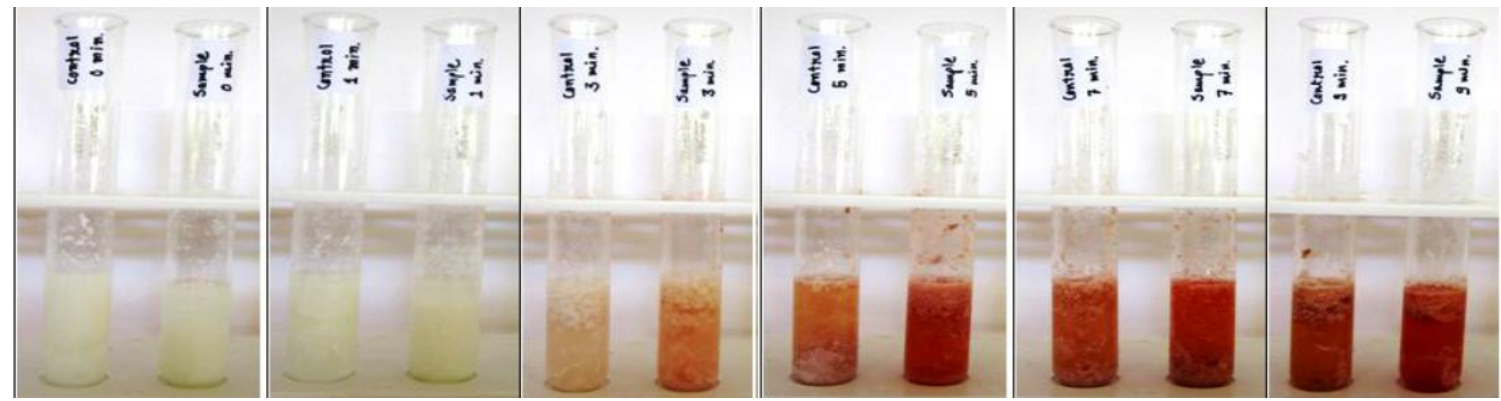

Fig.3 Effect of heating duration on Phenol Test for Ammonium sulphate
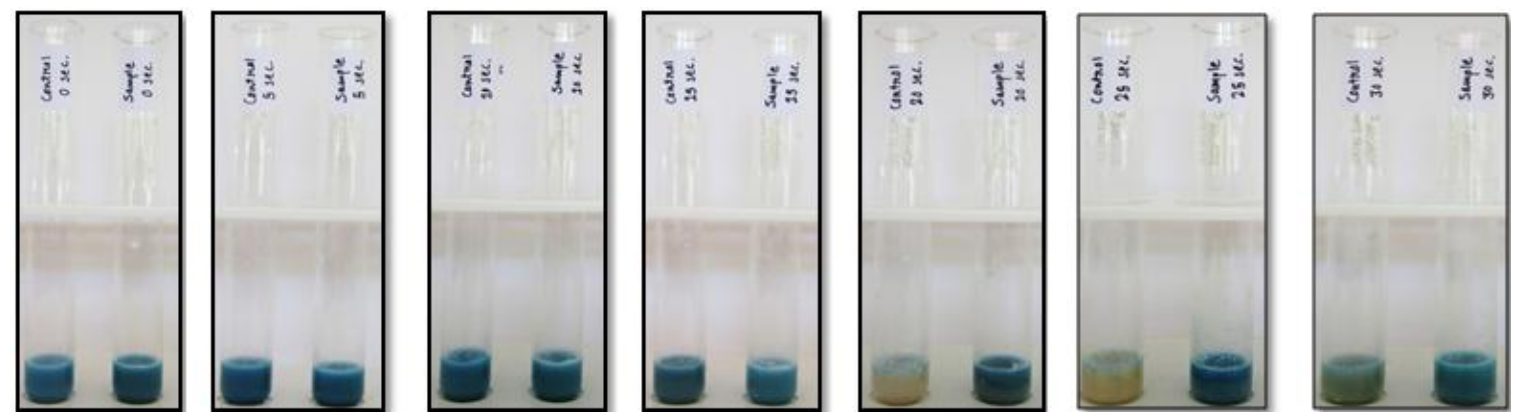

Fig.4 Effect of heating duration on Leach (heating on direct flame) for formaldehyde

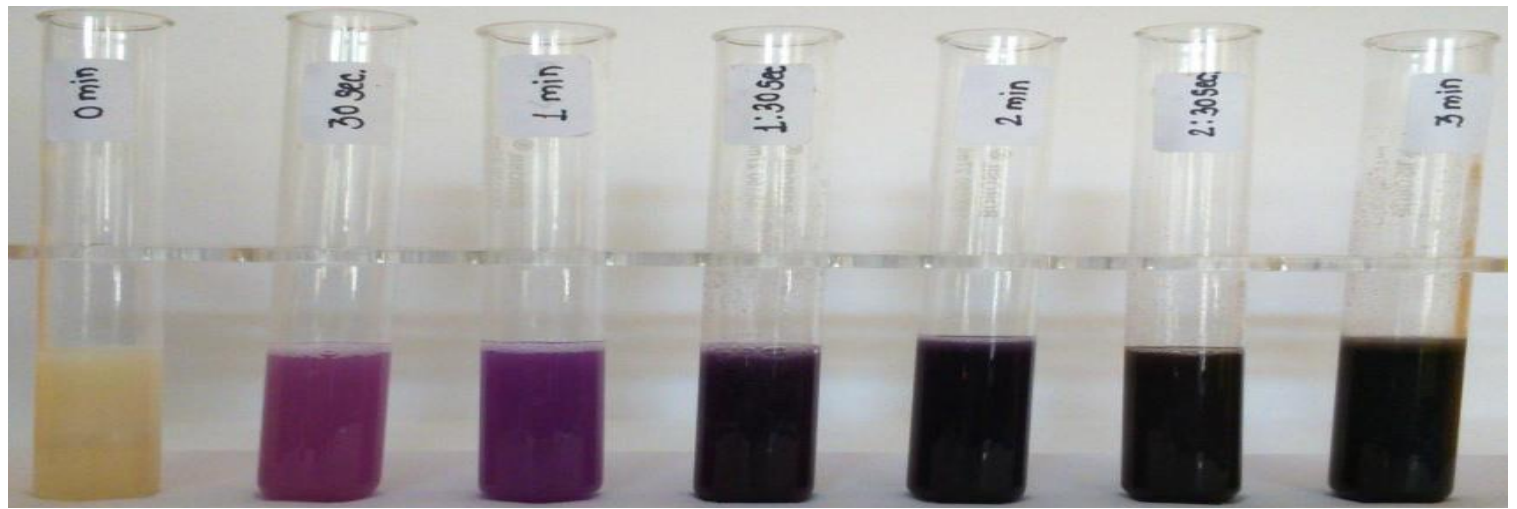


Fig.5 Effect of heating duration on Leach (heating in boiling water bath)

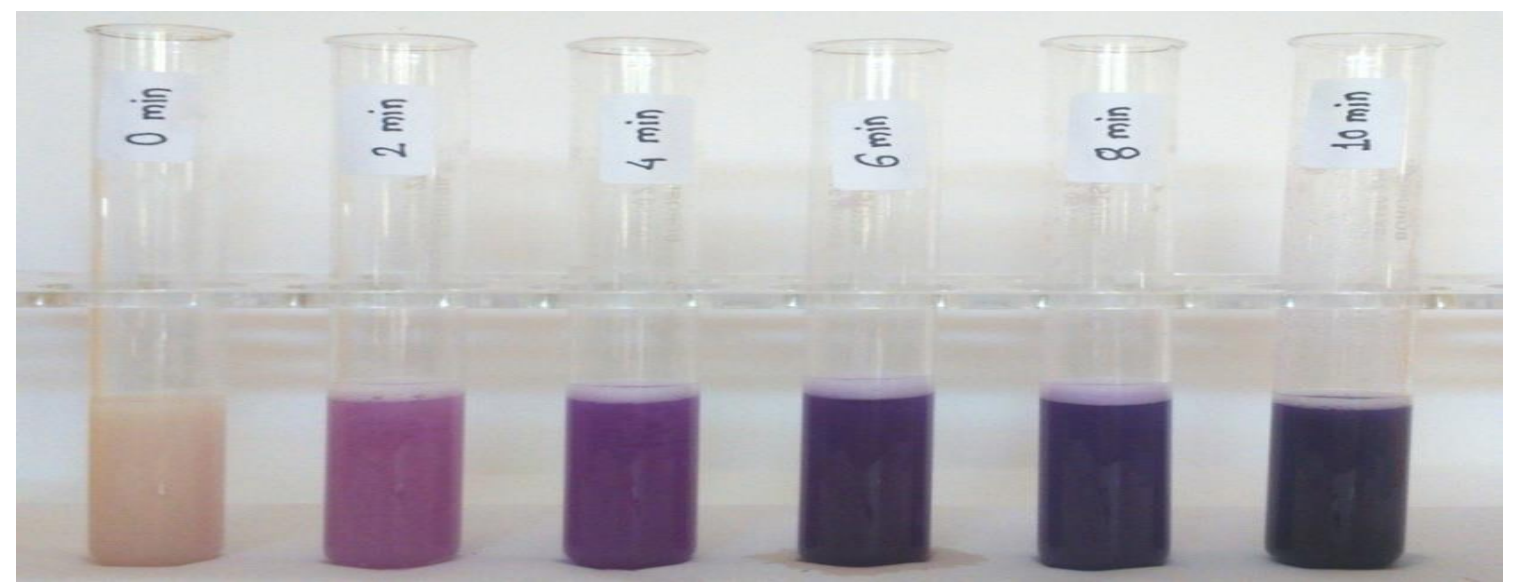

Reported procedure for phenol test uses the heating period of 20 seconds (Mittal and Roy, 1976; DGHS, 2005; Vishweshwar and Krishnaiah, 2005; NDDB, 2009; Srivastava, 2010 and FSSAI, 2012), which is in accordance with the result obtained in the present study.

Effect of heating on qualitative tests for detection of formaldehyde in milk

Leach test is one of the most methods for detection of added formaldehyde in milk. Leach test involve heating in a boiling water bath. Therefore, effect of duration of heating on performance of Leach test was evaluated. The samples of milk were prepared by adding formaldehyde at $0.006 \%$ level and effect of heating period was studied.

\section{Effect of heating on Leach test for formaldehyde}

Two different modes of heating are reported in the procedure for detection of formaldehyde in milk by Leach test. Williams and Sherman (1905), BIS (IS: 1961) and Vishweshwar and Krishnaiah (2005) suggested heating on direct flame whereas Sharma et al., (2012) suggested heating in boiling water bath. Therefore, work was carried out to evaluate effect of mode of heating and duration of heating on performance of Leach test for detection of formaldehyde in milk; which were prepared by adding 0.006 per cent formaldehyde.

\section{Leach test by heating on direct flame}

Leach test was performed using the prepared milk samples and the results are presented in figure 4 .

The result obtained in study suggested that heating on direct flame up to 30 seconds gives violet colour, but it disappeared on standing. On heating up to 1 minute gave dark violet colour which remained stable.

However, on further heating beyond 1 minute darkened the colour to brown, which was similar to that of the control sample.

Therefore, it can be concluded that the heating period of for 1 minute is optimum for getting optimum test results. On the other hand both under heating and overheating resulted in to false negative test.

\section{Leach test by heating in boiling water bath}

The results obtained for Leach test performed by heating the milk samples in boiling water bath are depicted in figure 5 . 
The result obtained in study suggested that on heating in boiling water bath up to 2 minutes, light violet colour appeared. On heating up to 4 minutes gave dark violet colour. However, on further heating up to 6 to 10 minutes, the desired violet colour turned to blue colour.

Considering the results obtained, heating time of 4 minutes in boiling water bath for Leach test can be suggested for optimum test results. Procedure for Leach test, as described by Sharma et al., (2012), suggested the heating of sample in water bath for 4 minutes, which is in accordance with the findings of present study.

Amongst the two different approaches (on direct flame and in boiling water bath) of heating in Leach test, the direct heating on flame found easy and faster, however, it necessitated carful heating on a small fame with intermittent mixing.

On the other hand, heating in boiling water bath is slightly lengthy and necessitates requirement of boiling water bath.

It can be concluded that the intensity of heating had significant effect in most of the tests used for the detection of adulteration in milk. Phenol test for detection of ammonium salts gave optimum results when heating time was limited to 20 seconds. Glucose was detected using Barfoed's test and it showed best differentiation between the adulterated and control sample when heating was carried out for 3 minutes.

Heating time for 5 minutes was found optimum for the detection of sucrose using Seliwanoff's test. Leach test for the detection of formaldehyde can be carried out either by heating the milk on direct flame or in water bath where the optimum result was found when the heating time was 1 minute and 4 minutes respectively.

\section{References}

Anon. 2017. National Dairy Development Board. Anand, India. http://www.nddb.org/information/stats/ milkprodindia. Cited 10/07/2017.

Arora S., Sharma V., Raj D., Ram M. and Kishore K. 2004. Status of milk adulteration in some states of North India. Ind. J. Dairy Sci., 57:65-66.

Barui A.K., Sharma R., Rajput Y. S. and Singh S. 2013. A rapid paper chromatographic method for detection of anionic detergent in milk. J. Food Sci. Technol., 50: 826.

BIS. 1960. Methods of test for dairy industry, Part- I: Rapid examination of milk, Bureau of Indian standard, Manak Bhavan, 9 Bahadur Shah Zafar Marg, New Delhi -110002.

DGHS. 2005. Manual of methods of analysis of foods (milk and milk products): Laboratory Manual-1. Directorate General of Health Services, Ministry of Health and Family Welfare, Government of India, New Delhi.

Filion D., Lavertu M., and Buschmann M. D. 2007. Ionization and Solubility of Chitosan Solutions Related to Thermosensitive Chitosan/GlycerolPhosphate Systems. Biomacromolecules, 8: 3224-3234.

FSSAI. 2012. Manual of methods of analysis of foods (milk and milk products) - A Laboratory Manual. Food safety and standards authority of India, Ministry of health and family welfare, Government of India, New Delhi.

Kamthania M., Saxena J., Saxena K. and Sharma D.K. (2014). Milk adulteration: Methods of detection \&remedial measures. Int. J. Engg. Tech. Res., 1: 15-20.

Mittal, S.B., and Roy, N. K. 1976. Rapid detection and estimation of extraneous ammonium sulphate in milk and effects 
of the added salt on certain physicochemical properties of buffalo milk. Indian J. Dairy Sci., 29(4): 283289.

NDDB, 2009. Methods for detection of common adulterants in milk and milk products. National Dairy Development Board. 83:1-36. http://www.dairyknowledge.in/sites/def ault/files/technews_83.pdf(09-07-2017).

Raynal, K., and Remeuf, F. 1998. The Effect of Heating on Physicochemical and Renneting Properties of Milk: A Comparison between Caprine, Ovine and Bovine Milk. Int.l Dairy J.8 (8): 695-706.

Roy N.K. and Mittal S.B. 1977. Rapid detection and estimation of extraneous glucose in milk and effects of the adulterant on certain physicchemicalproperties of buffalo milk. Indian J. Dairy Sc., 30(1): 30-35.

Sharma R., Rajput Y.S., Barui A.K. and Laxmana N.N. 2012. Detection of Adulterants in Milk - A Laboratory
Manual, Edition-1. NDRI Publication No: 88/2012. National Dairy research Institute, Karnal-132001.

Souza S.S., Cruz A.G., Walter E.H.M., Faria J.A.F., Celeghini R., Ferreira M., Granato D. and Sant'Ana A.S. 2011. Monitoring the authenticity of Brazilian UHT milk: a chemometric approach. Food Chem., 124: 692.

Srivastava M. K. 2010. Adulteration and detection of adulterants in milk. In "Handbook on analysis of milk Chemical and microbiological analysis of liquid milk". IBDC publishers, Lucknow, India. Pp. 95-155.

Vishweshwar K., and Krishnaiah N. 2005. Quality control of milk and processing. State Institute of Vocational Education, Director of Intermediate Education Government of Andhra Pradesh.

Williams, R.H., and Sherman H.C. 1905. The detection, determination, and rate of disappearance of formaldehyde in milk. J. Am. Chem. Soc., 27(12): 1497-1503.

\section{How to cite this article:}

Chaudhari Priyankaben Rameshbhai, Kakade Pooja Vilasrao, Jayswal Dharin, S.I. Patel, A.I. Shaikh and Aparnathi, K.D. 2017. Effect of Intensity of Heating on Qualitative Tests for the Detection of Common Adulterants in Milk. Int.J.Curr.Microbiol.App.Sci. 6(8): 2864-2871. doi: https://doi.org/10.20546/ijcmas.2017.608.342 